\title{
Expression of Epidermal Growth Factor-like Domain 7 is Increased by Transcatheter Arterial Embolization of Liver Tumors
}

\author{
Zhi Li ${ }^{1 \star}$, Cai-Fang Ni ${ }^{1 *}$, Jin Zhou ${ }^{2 \&}$, Xiao-Chun Shen ${ }^{3 \&}$, Yu Yin ${ }^{1}$, Peng Du ${ }^{1}$, Chao \\ Yang ${ }^{1}$
}

\begin{abstract}
Background: Epidermal growth factor-like domain multiple 7 (EGFL7), recently identified as a secreted protein regulated by oxygen exposure, plays a critical role in promoting metastasis of hepatocellular carcinoma (HCC). Transcatheter arterial embolization (TAE) is widely used for treatment of HCC, resulting in hypoxia in tumors and surrounding liver tissues. Accordingly, we proposed the hypothesis that there could be a relationship between expression of EGFL7 and response to TAE. Materials and Methods: We established a rabbit VX2 liver tumor model using percutaneous puncture technique guided by computed tomography. TAE and sham embolization were performed and the results were confirmed by MRI 3 weeks after inoculation. We investigated the EGFL7 expression of the two groups at $6 \mathrm{~h}$ and 3 days after intervention by means of immunohistochemistry and Western blotting. Results: Immunohistochemical staining demonstrated that the levels of EGFL7 protein significantly increased in the TAE-treated tumors compared with the control group at 6 hours $(P=0.031)$ and 3 days $(P=0.020)$ after intervention. Meanwhile, the relative EGFL7 protein detected in TAE group also up-regulated compared with the control group at 6 hours $(P=0.020)$ and 3 days $(P=0.024)$ after intervention. Conclusions: This study reveals an increase of EGFL7 expression in rabbit VX2 liver tumors after TAE. The role of EGFL7 in HCC, especially its biological behavior after TAE, needs further investigation.
\end{abstract}

Keywords: VX2 rabbit liver tumor - Transcatheter arterial embolization- EGFL7

Asian Pac J Cancer Prev, 16 (3), 1191-1196

\section{Introduction}

Hepatocellular carcinoma (HCC) is the sixth most common cancer and the third leading cause of cancer death in the world, resulting in more than 600,000 deaths each year (Jemal et al., 2011), which is characterized by comorbidity of cirrhosis that is induced by viral hepatitis, alcohol, and nonalcoholic steatohepatitis (Abdelaziz et al., 2014). Most patients with HCC are diagnosed at advanced stages, while curative treatments such as hepatic resection and liver transplantation are not feasible as it can be expected (Forner et al., 2012). Transcatheter arterial chemoembolization (TACE) are widely used palliative treatment for patients with unresectable HCC and have shown encouraging results in survival (Xu et al., 2014; Llovet and Bruix, 2003; Mao et al., 2012; Dai et al., 2013). However, it is generally considered that TACE rarely achieves total necrosis to the targeted liver tumor(Sasaki et al., 2006) and some reports even suggest that TACE may actually accelerate extrahepatic metastasis (Liou et al., 1995).

Recent studies have shed light on the implications of hypoxia induced by TACE (due to transcatheter arterial embolization, TAE) in liver tumors (Lai et al., 2014). Increased vascular endothelia growth factor (VEGF) were reported in liver tumors after TAE, which stimulates angiogenesis in the residual viable tumor (Liu et al., 2008; Shim et al., 2008; Hsieh et al., 2011). The importance of knowing this molecular mechanism is underscored by the positive results of the START study (Chung et al., 2013; Han et al., 2013), which combined sorafenib with TACE in the treatment of HCC. However, metastasis of HCC is a multifactorial, multistep, and complex process (Farazi and DePinho, 2006). It is reasonable to believe that there would be other pro-metastatic factors unregulated after TAE besides VEGF.

Epidermal growth factor-like domain multiple 7 (EGFL7) is a secreted protein that contains two EGFlike domains and is conserved across species (Nichol and Stuhlmann, 2012; Xu et al., 2014). It was initially regarded as an endothelial cell-specific gene and an important regulator in tubulogenesis during embryonic development (Parker et al., 2004). However, recent studies have demonstrated that EGFL7 expresses in several

${ }^{1}$ Department of Interventional Radiology, ${ }^{2}$ Department of General Surgery, ${ }^{3}$ Department of Respiratory Medicine, the First Affiliated Hospital of Soochow University, Suzhou, Jiangsu, China ${ }^{\circledR}$ Equal contributors *For correspondence: szncf003@hotmail.com 
tumors and many cancer cell lines (Fan et al., 2013). Nichol et al. (2012) reported that the elevated levels of EGFL7 expression in tumors and its possible role in promoting tumor angiogenesis make EGFL7 a potential target for antiangiogenesis therapy. In addition to its role in tumor angiogenesis, EGFL7 has been shown to enhance intrahepatic and pulmonary metastasis of HCC cells by promoting cell motility by EGF receptor-dependent phosphorylation of focal adhesion kinase (Wu et al., 2009).

Hypoxia has been reported to up-regulate the expression of EGFL7 in human coronary artery endothelial cells (Badiwala et al., 2010). Xu et al. (2008) reported that EGFL7 gene expression was significantly decreased in the neonatal rat lungs after hyperoxic exposure. These studies seem to indicate that the expression of EGFL7 is regulated by oxygen exposure. TAE inevitably results in a hypoxic insult to $\mathrm{HCC}$ and surrounding liver tissue. Hence, we proposed the hypothesis that there could be a ralationship between expression of EGFL7 and TAE. To test this hypothesis, we investigated the change of EGFL7 expression in the rabbit VX2 liver tumor after TAE.

\section{Materials and Methods}

Animal care procedures and experimental protocol were approved by institutional animal care and use committee based on institutional guidelines. Adult New Zealand white rabbits weighing 4-5 kg were used in this study. Fasting was prescribed the night before inoculation, imaging examination and TAE treatment. And 3\% pentobarbital was used for general anesthesia via ear vein at a dose of $30 \mathrm{mg} / \mathrm{kg}$.

\section{Animal model and tumor implantation}

Rabbit VX2 carcinoma preparation was performed as previously described (Parvinian et al., 2014; Sun et al., 2014). VX2 cells were initially implanted in both hind limbs of donor rabbits. After 3 weeks of tumor growth, tumors in hind limb were collected and small tumor fragments (approximately $1.0 \mathrm{~mm}^{3}, 2 \times 10^{5}$ tumor cells) were dissected from the viable tumor tissues, which were soaked in saline until use.

Experimental rabbits were anesthetized and fixed in a supine position and skin on the upper abdomen was prepared by shaving and sterilization. Then a $17 \mathrm{G}$ coaxial introducer needle (MCXS1810BP, Angiotech, Flordia) was inserted percutaneously into the left lobe of the liver using CT-guided (Brilliance 16, Philips, Cleveland) method. Afterwards a core needle of the trocar was used to coaxially push the 2 tumor explants into the target site through the sheathed needle, with pieces of Gelfoam $(0.5$ $\mathrm{cm}$; Jinling Pharmaceutical Company, Nanjing) filling the needle tract in the end.

\section{Magnetic resonance imaging}

To detect the formation and size of VX2 liver tumor, magnetic resonance imaging (MRI) was performed with a 1.5-T unit (Siemens Medical Solutions, Erlangen, Germany) 3 weeks after tumor implantation. The protocols consisted of T1WI, T2WI and gadolinium-enhanced T1WI with slice thickness: $3 \mathrm{~mm}$, matrix: $128 \times 128$, FOV
$24 \mathrm{~cm} \times 24 \mathrm{~cm}, \mathrm{NEX}=4$. Gadolinium-enhanced T1WI was acquired after $0.1 \mathrm{mmol} / \mathrm{kg}$ body weight gadopentetate dimeglu-mine (Magnevist; Bayer, Berlin, Germany) was administered to each rabbit via the ear vein by means of manual fast bolus injection. Tumor growth was considered positive when tumors were identified in axial imaging planes by two independent MRI specialists.

\section{Experimental grouping}

Rabbit models of VX2 liver tumors were established and divided into two groups. In the TAE group, animals were subjected to hepatic artery embolization with lipiodol. In the control group, animals underwent sham embolization with saline. Animals in each group were randomly assigned into two subgroups on the basis of timing of sacrifice: 6 hours and 3 days after experimental intervention. At each time points, 9 animals were sacrificed for immunohistochemical staining $(n=5)$ and western blotting $(n=4)$.

\section{Transcatheter arterial embolization}

TAE was performed on all rabbits by 2 researchers under digital subtraction angiography (DSA). After being anesthetized as described above, the right femoral artery of the rabbit was exposed, an 4-F sheath (Groshong* NXT ClearVue*, Bard, USA) was inserted by Seldinger technique. The micro catheter (Progreat, TERUMO, Japan) was selectively inserted into the left hepatic artery. Angiography (flow rate $1 \mathrm{ml} / \mathrm{s}$, total volume $3 \mathrm{ml}$ ) was performed via the microcatheter. The tumor stain with tumor feeding arteries in the left hepatic lobe was readily visualized.

Lipiodol (Guerbet, France) was used as our embolic agent for TAE, which was superselectively delivered by hand injection through the same catheter previously placed under fluoroscopy. The embolization endpoint was complete stasis of antegrade blood flow(Lewandowski et al., 2007). For the control group, $3 \mathrm{ml}$ of saline was injected instead of the lipiodol.

\section{Immunohistochemistry}

Five rabbits in each groups were sacrificed at each time points (6hours, 3days) after intervention with an intravenous injection of $150-200 \mathrm{mg} / \mathrm{kg}$ pentobarbital. Tumor tissue specimens, including the largest diameter of tumor and a small quantity of peritumoral liver tissue were collected, fixed in $10 \%$ neutral buffered formalin, and embedded in paraffin. An $4-\mu \mathrm{m}$ thick slice was cut and processed for staining with anti-EGFL7 polyclonal antibody (Santa Cruz Biotechnology, 1:150 dilution) according to the manufacturer's instruction.

The immunohistochemical staining was evaluated by two pathologists in a blinded manner. All slides were examined under a light microscope throughout the entire section. Discrepancies were resolved on the conference microscope. The percentage of positive staining cells was measured by Image Process System(HPISA-1000; Qianping Image Technology Limited Company, China). The staining was graded on a 4-point scale according to the percentage of positive cancer cells(Wu et al.,2009) : grade 0 ( $\leq 10 \%$ positive); grade $1+(11 \%$ to $25 \%$ positive); grade 


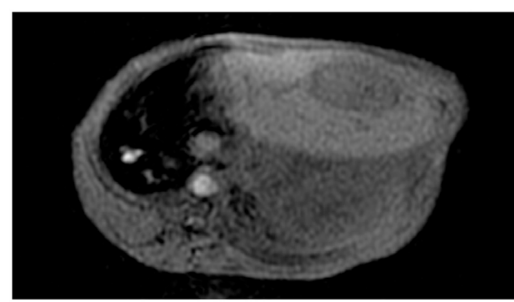

B
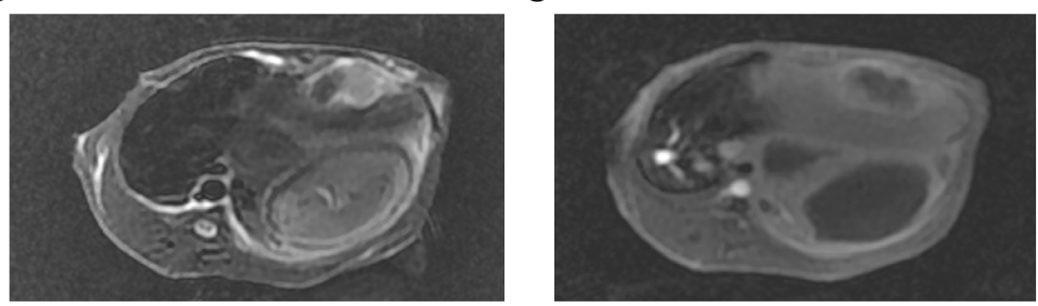

Figure 1. MRI of Rabbit Liver 3w after Tumor Implantation. A, Low signal on T1WI. B, High signal on T2WI. C, Enhanced scan shows significantly enhanced lesions in the peripheral portion of the tumor

A

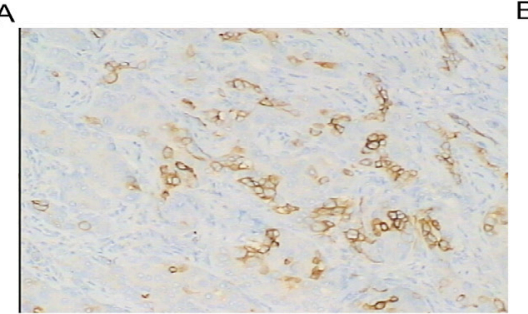

B

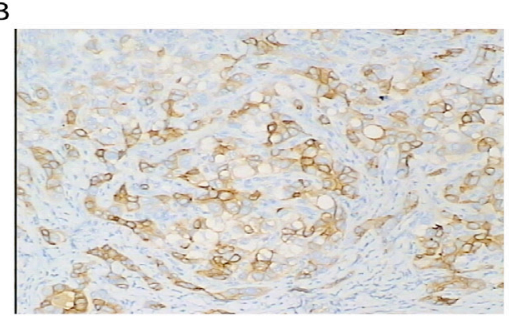

C

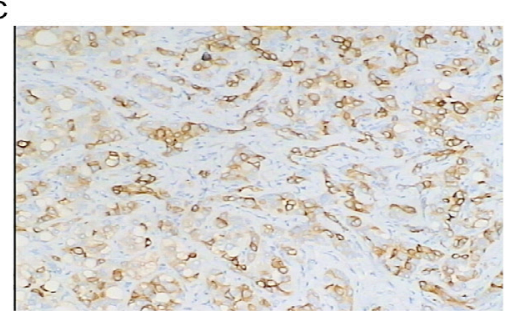

Figure 3. Immunohistochemical Staining for EGFL7 $(\times \mathbf{1 0 0})$. The staining was graded using a 4-point scale according to the percentage of positive tumor cells. A: Representative image of control group at $6 \mathrm{~h}$ after intervention, EGFL 7 expression is seen in $11 \%-25 \%$ of cancer cells (scored as 1+). B: Representative image of TAE group at $3 \mathrm{~d}$ after intervention, EGFL7 expression is seen in 25\%-50\% of cancer cells (scored as 2+). C: Representative image of TAE group at $3 \mathrm{~d}$ after intervention, EGFL7 expression is seen more than $51 \%$ cancer cells (scored as $3+$ )

\section{$2+(26 \%$ to $50 \%$ positive); grade $3+(\geq 51 \%$ positive $)$.}

\section{Western blot}

Four rabbits in each groups were sacrificed the same way as described above. Viable VX2 tumor were rapidly dissected and extracted, and the expression of EGFL7 were measured by western blot as described by Fan et al(Fan et al., 2013). Briefly, SDS-PAGE (5\% stacking gel and $12 \%$ separating gel) was used to separate $100 \mu \mathrm{g}$ of total protein. Proteins were transferred to polyvinylidene difluoride membrane (Millipore, Bedford, MA). Goat anti-rabbit EGFL7 polyclonal antibody (SantaCruz Biotechnology, Santa Cruz, CA, 1:150) was used as the primary antibody. Horseradish peroxidase- conjugated Mouse anti-goat secondary antibody (KPL, Gaithersburg, MD; 1:6000) was used as secondary antibody. Beta-actin protein was also determined by using the specific antibody (Sigma, St Louis, MO) as a loading control. Antigenantibody complexes were visualized by the enhanced chemiluminescence (ECL) system (Pierce, Rockford, IL).

\section{Data analysis}

Statistical analyses were carried out by SPSS version 13.0 (SPSS, Chicago, IL). The Student t-test was used to compare tumor diameter and western bloting results between the TAE group and control group. The Mann-Whitney U-test was used to compare the immunohistochemistry results between the two groups. A $P$ value of $<0.05$ was considered statistically significant.

\section{Results}

\section{Animal model}

All 46 rabbits underwent puncture and successful VX2 liver tumor model in the left liver lobe were achieved in 41
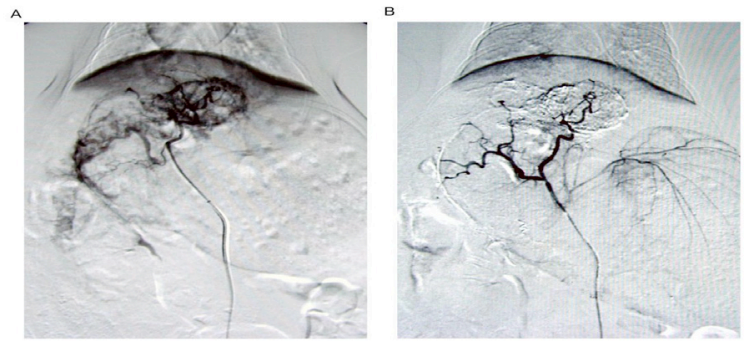

Figure 2. Representative Rabbit Hepatic Arteriograms Transcatheter arterial embolization. The peripheral portion of the tumor is hypervascular prior to embolization (a). After embolization (b) there is abrupt cut off of the feeding artery without any remaining peripheral hypervascularity

cases, with a rate of $89.1 \%(41 / 46)$ which was confirmed 3 weeks after inoculation by MRI (Figure 1). Among the 5 failed cases, 1 died from anesthesia during MRI imaging, 1 case with tumor growth outside liver, and 3 other were found no tumor growth. The maximum tumor diameter of the TAE group and control group were $1.61 \pm 0.25 \mathrm{~cm}$ and $1.54 \pm 0.26 \mathrm{~cm}$ respectively, without significant differences $(P=0.439)$.

TAE was nicely performed in 38 VX2 implanted models, with a technical success rate of $92.7 \%$ (38/41). Failure of femoral artery puncture happened to 3 cases. VX2 liver tumors were visualized as a region of hypervascular supply at angiography (Figure 2a), which showed typically more intense in the peripheral portion of the tumor. In TAE group, 2-4.5ml lipiodol (mean, 3.4ml) was delivered through microcatheter in hepatic artery. Post-TAE angiography confirmed the successful reduction in blood flow to tumors (Figure 2b). In control group, $3 \mathrm{ml}$ normal saline was injected with the same manner as in TAE group. In TAE group, 2 rabbits did not survive 3 day 
Table 1. Comparisons of EGFL7 Protein Levels between Groups at Different Time Points

\begin{tabular}{|c|c|c|c|c|c|c|}
\hline \multirow[t]{2}{*}{ Time } & \multirow{2}{*}{ Group } & \multicolumn{4}{|c|}{ EGFL7 protein level } & \multirow[t]{2}{*}{$\mathrm{P}$} \\
\hline & & 0 & $1+$ & $2+$ & $3+$ & \\
\hline \multirow[t]{2}{*}{$6 \mathrm{~h}$} & TAE $(n=5)$ & 0 & 0 & 3 & 2 & \multirow[t]{2}{*}{0.031} \\
\hline & Control(n=5) & 0 & 3 & 2 & 0 & \\
\hline \multirow[t]{2}{*}{$3 d$} & $\operatorname{TAE}(n=5)$ & 0 & 0 & 2 & 3 & \multirow[t]{2}{*}{0.020} \\
\hline & $\operatorname{Control}(n=5)$ & 0 & 3 & 2 & 0 & \\
\hline \multirow[t]{2}{*}{ Total } & $\operatorname{TAE}(\mathrm{n}=10)$ & 0 & 0 & 5 & 5 & \multirow[t]{2}{*}{0.001} \\
\hline & $\operatorname{Control}(\mathrm{n}=10)$ & 0 & 6 & 4 & 0 & \\
\hline
\end{tabular}

A

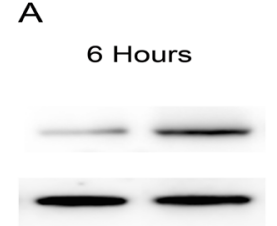

Control TAE

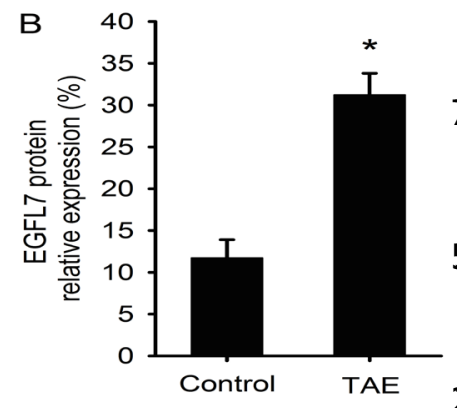

C

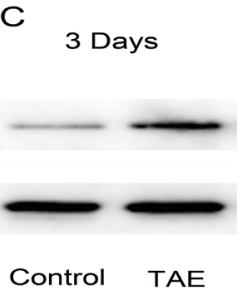

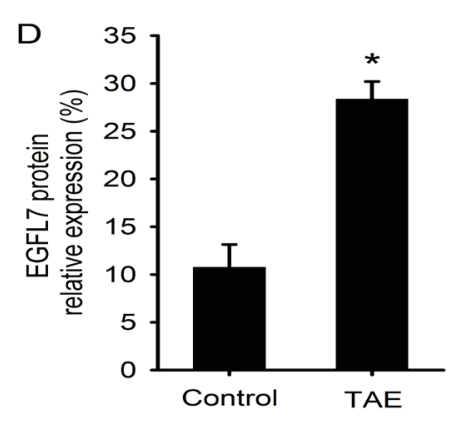

Figure 4. Western blot test of EGFL7 expression $6 \mathrm{~h}$ (A) and 3 days (B) after intervention. The relative EGFL7 protein expression in TAE group is significantly higher compared with the control group $6 \mathrm{~h}$ after intervention $(\mathrm{B}, * \mathrm{P}=0.020)$. And the same result was observed at 3 days after intervention $(\mathrm{D}, * \mathrm{P}=0.024)$

after the procedure. A total of 36 rabbit models (18 in each group) were included in the final analysis.

\section{Expression of EGFL7 in VX2 tumor}

Positive staining of EGFL7 was detected mainly in cytoplasm of the viable tumor cells that were distributed at the periphery of necrotic tumor regions, and weak EGFL7 expression could also be found in vascular endothelial cells. The immunohistochemical staining was graded on 4-point scale according to the percentage of positive tumor cells. The levels of EGFL7 expression were significantly higher in the TAE-treated tumors compare with those in the control group at each observation time points (Figure 3 , Table 1).

We also deployed western blot to analyze the expression of EGFL 7 protein in VX2 tumor tissues (Figure 4). At 6h after intervention, the EGFL7 protein level in TAE group were significantly higher compared with the control group $(0.313 \pm 0.026$ vs $0.118 \pm 0.022$, $P=0.020$ ). And the similar results were observed at 3 days (0.283 \pm 0.019 vs $0.108 \pm 0.024, P=0.024)$.

\section{Discussion}

In this study, we successfully build the rabbit VX2 liver tumor models which were confirmed by MRI. Then, TAE and sham embolization were performed in these liver tumor models. Immunohistochemistry and western blot were deployed to detect the expression of EGFL7 protein at $6 \mathrm{~h}$ and 3 days after intervention. A significant increase in EGFL7 protein levels were observed in the TAE-treated tumors compared with tumors in control group at each time point. These data suggested that overexpression of EGFL7 is a common event in VX2 tumors treated with TAE.

The likely mechanism for the up-regulation of EGFL7 after embolization is through hypoxia and ischemia of the residual viable tumor tissues. Badiwala et al(Badiwala et al., 2010) reported that hypoxia up-regulates human coronary artery endothelial cells expression of EGFL7. Xu et al(Xu et al., 2008) reported that EGFL7 gene expression was significantly decreased in the neonatal rat lungs after hyperoxic exposure. These studies seem to indicate that the expression of EGFL7 is regulated by oxygen exposure. The aim of TAE for liver tumors is to induce ischemia and hypoxia within the tumor and subsequent tumor necrosis. However, due to the complexity of blood supply for liver tumors and the limitations of the TAE technique, embolization of the tumor feeding vessel is incomplete, which allows part of the tumor to survive in hypoxic microenvironment. Hence, we assumed that the overexpression of EGFL 7 in VX2 tumors after TAE was most likely resulted from intratumoral hypoxia generated by the procedure.

EGFL 7 was initially characterized as a gene specifically expressed by blood vessel endothelial cells in normal organs during development and in the adult (Soncin et al., 2003; Lelievre et al., 2008). However, this specificity of expression has been lost in cancer since EGFL7 has been detected in tumor cells themselves, in addition to endothelial cells. The expression level was linearly correlated with tumor differentiation in glioma (Huang et al., 2010) and colon cancer (Diaz et al., 2008), and the higher expression were associated with poorer prognosis and higher metastatic score in HCC patients (Wu et al., 2009). All these observations strongly suggested a role for EGFL7 in tumor growth and metastasis.

The possible mechanisms of EGFL7 in promoting tumor progression have been reported previously (Delfortrie et al., 2011; Nichol and Stuhlmann, 2012). In gliomas, EGFL7 expression correlates with increased cell proliferation and microvascular density(Huang et al., 2010). Injection of mice with HCC cells that express knockdown levels of EGFL7 results in the formation of smaller tumors that exhibit reduced vascular density compared with the tumors formed in mice that were injected with control cells(Wu et al., 2009). These studies support that EGFL7 is a possible pro-angiogenesis factor. In addition to its role in tumor angiogenesis, EGFL7 has been showed to enhance HCC cell motility through EGF receptor-dependent phosphorylation of focal adhesion kinase (Wu et al., 2009). What's more, Delfortrie et al (2011) reported that EGFL7 promotes tumor escape 
Expression of Epidermal Growth Factor-like Domain 7 is Increased by Transcatheter Arterial Embolization of Liver Tumors

from immunity by reducing the expression of endothelial molecules that mediate immune cell infiltration. All these findings above highlight the promising role of EGFL7 as a therapeutic target to fight cancer, including HCC. This study reveals an increase of EGFL7 expression in rabbit VX2 liver tumor after TAE. The association between overexpression of EGFL7 and tumor metastases is definitely worth exploring in the future.

This study had some limitations though the research done was carefully designed and performed. Firstly, the study indicated that TAE promote the up-regulation of EGFL7 expression in rabbit VX2 tumor, however, the relation with metastasis has not yet been proven. Secondly, we did not assess alternative techniques that are more common used in clinical practice, such as chemoembolization or radioembolization. Finally, we adopted immunohistochemistry and western blot to analysize the expression of EGFL7 but failed to verify the expression with other quantitative genetical methods, such as polymerase chain reaction (PCR) or quantitative real-time PCR. The up-coming study for us is to evaluate dynamical changes in plasma of EGFL 7 after TACE and the association with tumor metastasis and prognosis.

In conclusion, this study indicates that the expression of EGFL7 is up-regulated in liver tumors after TAE. The likely mechanism for this is intratumoral hypoxia caused by the procedure. Over-expression of EGFL7 could be a cause for tumor metastasis associated with some failed treatment. Studies ahead would be conducted to confirm similar results in clinical patients. Targeting EGFL7 could represent a potential therapeutic strategy in combination with TAE and/or TACE for the treatment of HCC.

\section{Acknowledgements}

This study was supported by the Science and Technology Bureau and Sanitary Bureau of Suzhou (KJXW2013003) \& (KJXW2011008), Soochow University (SDY2013A26), National Science Foundation of China (No.81302148), Natural Science Research Foundation of Colleges and Universities in Jiangsu Province (13KJB320019), Science Foundation of Postgraduate Cultivation and Innovation Project of Jiangsu Province (CXZZ12_0837)

\section{References}

Abdelaziz AO, Elbaz TM, Shousha HI, et al (2014). Survival and prognostic factors for hepatocellular carcinoma: an Egyptian multidisciplinary clinic experience. Asian Pac J Cancer Prev, 15, 3915-20.

Badiwala MV, Tumiati LC, Joseph JM, et al (2010). Epidermal growth factor-like domain 7 suppresses intercellular adhesion molecule 1 expression in response to hypoxia/reoxygenation injury in human coronary artery endothelial cells. Circulation, 122, 156-61.

Chung YH, Han G, Yoon JH, et al (2013). Interim analysis of START: Study in Asia of the combination of TACE (transcatheter arterial chemoembolization) with sorafenib in patients with hepatocellular carcinoma trial. Int J Cancer, 132, 2448-58.

Dai XZ, Yin HT, Sun LF, et al (2013).Potential therapeutic efficacy of curcumin in liver cancer. Asian Pac J Cancer Prev, 14, 3855-9.

Delfortrie S, Pinte S, Mattot V, et al (2011). Egfl7 promotes tumor escape from immunity by repressing endothelial cell activation. Cancer Res, 71, 7176-86.

Diaz R, Silva J, Garcia JM, et al (2008). Deregulated expression of miR-106a predicts survival in human colon cancer patients. Genes Chromosomes Cancer, 47, 794-802.

Fan C, Yang LY, Wu F, et al (2013). The expression of Egfl7 in human normal tissues and epithelial tumors. Int J Biol Markers, 28, 71-83.

Farazi PA, DePinho RA (2006). Hepatocellular carcinoma pathogenesis: from genes to environment. Nat Rev Cancer, 6, 674-87.

Forner A, Llovet JM, Bruix J (2012). Hepatocellular carcinoma. Lancet, 379, 1245-55.

Han G, Yang J, Shao G, et al (2013). Sorafenib in combination with transarterial chemoembolization in Chinese patients with hepatocellular carcinoma: a subgroup interim analysis of the START trial. Future Oncol, 9, 403-10.

Hsieh MY, Lin ZY, Chuang WL (2011). Serial serum VEGF-A, angiopoietin-2, and endostatin measurements in cirrhotic patients with hepatocellular carcinoma treated by transcatheter arterial chemoembolization. Kaohsiung J Med Sci, 27, 314-22.

Huang CH, Li XJ, Zhou YZ, et al (2010). Expression and clinical significance of EGFL7 in malignant glioma. $J$ Cancer Res Clin Oncol, 136, 1737-43.

Jemal A, Bray F, Center MM, et al (2011). Global cancer statistics. CA Cancer J Clin, 61, 69-90.

Lai JP, Conley A, Knudsen BS, et al (2014). Hypoxia after transarterial chemoembolization (TACE) may trigger a progenitor cell phenotype in hepatocellular carcinoma (HCC). Histopathology.

Lelievre E, Hinek A, Lupu F, et al (2008). VE-statin/egfl7 regulates vascular elastogenesis by interacting with lysyl oxidases. Embo J, 27, 1658-70.

Lewandowski RJ, Wang D, Gehl J, et al (2007). A comparison of chemoembolization endpoints using angiographic versus transcatheter intraarterial perfusion/MR imaging monitoring. $J$ Vasc Interv Radiol, 18, 1249-57.

Liou TC, Shih SC, Kao CR, et al (1995). Pulmonary metastasis of hepatocellular carcinoma associated with transarterial chemoembolization. J Hepatol, 23, 563-8.

Liu WG, Gu WZ, Zhou YB, et al (2008). The prognostic relevance of preoperative transcatheter arterial chemoembolization (TACE) and PCNA/VEGF expression in patients with Wilms' tumour. Eur J Clin Invest, 38, 931-38.

Llovet JM, Bruix J (2003). Systematic review of randomized trials for unresectable hepatocellular carcinoma: Chemoembolization improves survival. Hepatology, 37, 429-42.

Mao YM, Luo ZY, Li B, et al (2012). Prospective study on the survival of HCC patients treated with transcatheter arterial lipiodol chemoembolization. Asian Pac J 
Cancer Prev, 13, 1039-42.

Nichol D, Stuhlmann H (2012). EGFL7: a unique angiogenic signaling factor in vascular development and disease. Blood, 119, 1345-52.

Parker LH, Schmidt M, Jin SW, et al (2004). The endothelial-cell-derived secreted factor Egfl7 regulates vascular tube formation. Nature, 428, 754-8.

Parvinian A, Casadaban LC, Gaba RC (2014). Development, growth, propagation, and angiographic utilization of the rabbit VX2 model of liver cancer: a pictorial primer and "how to" guide. Diagn Interv Radiol, 20, 335-40.

Sasaki A, Iwashita Y, Shibata K, et al (2006). Preoperative transcatheter arterial chemoembolization reduces longterm survival rate after hepatic resection for resectable hepatocellular carcinoma. Eur J Surg Oncol, 32, 773-9.

Shim JH, Park JW, Kim JH, et al (2008). Association between increment of serum VEGF level and prognosis after transcatheter arterial chemoembolization in hepatocellular carcinoma patients. Cancer Sci, 99, 2037-44.

Soncin F, Mattot V, Lionneton F, et al (2003). VE-statin, an endothelial repressor of smooth muscle cell migration. Embo J, 22, 5700-11.

Sun YX, Cheng W, Han X, et al (2014). In vivo experimental study on the effects of fluid in increasing the efficiency of radiofrequency ablation. Asian Pac J Cancer Prev, 15, 5799-804.

Wu F, Yang LY, Li YF, et al (2009). Novel role for epidermal growth factor-like domain 7 in metastasis of human hepatocellular carcinoma. Hepatology, 50, 1839-50.

Xu C, Lv PH, Huang XE, et al (2014). Safety and efficacy of sequential transcatheter arterial chemoembolization and portal vein embolization prior to major hepatectomy for patients with HCC. Asian Pac J Cancer Prev, 15, 703-6.

Xu D, Perez RE, Ekekezie II, et al (2008). Epidermal growth factor-like domain 7 protects endothelial cells from hyperoxia-induced cell death. Am J Physiol Lung Cell Mol Physiol, 294, 17-23.

Xu HF, Chen L, Liu XD, et al (2014). Targeting EGFL7 expression through RNA interference suppresses renal cell carcinoma growth by inhibiting angiogenesis. Asian Pac J Cancer Prev, 15, 3045-50. 